\title{
Modelo para evaluar los efectos de los procesos de acreditación con el SINAES en una carrera de una universidad estatal costarricense: aportes para su creación
}

\section{A model to evaluate accreditation processes effects with SINAES Department in a career of a Costa Rican state university: set contributions}

\author{
Lorena Somarribas Meza ${ }^{1}$ \\ Instituto Tecnológico de Costa Rica \\ Cartago, Costa Rica \\ Isomarribasmeza@gmail.com \\ Maricel Araya Matamoros ${ }^{2}$ \\ Instituto Tecnológico de Costa Rica \\ Cartago, Costa Rica \\ marayamatamoros@gmail.com
}

Recibido 21 setiembre 2011 * Aceptado 05 octubre 2011 * Corregido 13 diciembre 2011

Resumen. Algunos estudios señalan que la consolidación de los procesos de acreditación como mecanismos de aseguramiento de la calidad, hacen necesario que se creen instrumentos para medir su impacto efectivo sobre la calidad. En este contexto se desarrolla la investigación con el interés de generar aportes para la creación de un modelo de evaluación de los efectos de los procesos de acreditación realizados ante el SINAES. Por "efecto" se entiende todo comportamiento o acontecimiento -fuera de la línea de gestión institucional-, del cual puede razonablemente decirse que ha sido influido por algún aspecto de la acreditación. Con ese fin se eligió un referente de calidad en educación superior a partir de propuestas de la UNESCO, el cual involucra los principios de relevancia, pertinencia, equidad, eficiencia y eficacia, desde una perspectiva vigente que conceptualiza a la educación como un derecho humano. Este se denominó "Constructo Calidad". Además, se analizó su concordancia con el nuevo Modelo de acreditación del SINAES, determinando que ese último está acorde con esta conceptualización. Paralelamente se eligió la metodología de medición de efectos o impactos de la GTZ, Agencia de Cooperación Técnica

\footnotetext{
${ }^{1}$ Máster en Administración Universitaria de la Universidad de Costa Rica. Licenciada en Administración de Empresas del Instituto Tecnológico de Costa Rica. Licenciada en Administración de Empresas con énfasis en Contabilidad de la Universidad Estatal a Distancia. Actualmente labora en la Auditoría Interna del Instituto Tecnológico de Costa Rica, destacada en el área de Auditoría Operativa.

${ }^{2}$ Máster en Administración Universitaria de la Universidad de Costa Rica. Licenciada en Administración de Empresas con énfasis en Contaduría Pública del Instituto Tecnológico de Costa Rica. Actualmente labora como Auditora en el Instituto Tecnológico de Costa Rica, destacada en autoevaluación y validación de la actividad de la Auditoría Interna.
} 
Revista Gestión de la Educación, Vol.1, N², pp.35-66, ISSN 2215-2288, julio-diciembre, 2011

Alemana. También, se señalaron algunos efectos que podrían esperarse al aplicar criterios concretos del Modelo SINAES y se eligió un criterio para delinear una idea preliminar del Modelo de Evaluación de Efectos.

Palabras clave. Calidad de la educación, acreditación, evaluación, pertinencia.

\begin{abstract}
Some studies point out accreditation processes as mechanisms to guarantee quality developing instruments to evaluate the effective impact in quality. In this context, this research gives contributions to set a model to assess effects of accreditation processes made to SINAES. For this study, "effect" is understood as the behavior or event out of the institutional management from which it is reasonable to believe that have been influenced by some aspect of accreditation. For this purpose a a standard of quality on higher educational levels was selected based on UNESCO's proposals, this involves principles of relevance, pertinence, efficiency and effectiveness, from an updated perspective which conceptualizes education as a human right. This was called "Construct Quality". In addition, it was analyzed its concordance with the new accreditation model from SINAES, and it was stated that this model is consistent with this conceptualization. Besides, methodology of the German Technical Cooperation Agency (GTZ) was designated for assessing effects or impacts. Moreover, some effects can be expected from application of specific criteria of the model SINAES. Furthermore, researchers chose criteria to define a preliminary idea of the impact of this Effect Assessment Model.
\end{abstract}

Key words. Educational quality, accreditation, evaluation, assessment, relevance, pertinence.

\title{
Introducción
}

Durante varios siglos la sociedad no cuestionaba ni dudaba de la calidad de las universidades; se daba por un hecho su existencia. Sin embargo, en las últimas décadas los temas de la calidad y su aseguramiento, mediante la evaluación, tienen una importancia preponderante para las universidades. La motivación para esta búsqueda del fortalecimiento de la calidad se encuentra en diversas razones, entre estas: fuerte crecimiento de la demanda, masificación de la enseñanza superior, surgimiento de nuevas instituciones que no se acompañó de mecanismos para asegurar la calidad, a las que se agregan ahora presiones externas relacionadas con el papel más importante que desempeñan los establecimientos de enseñanza superior en el desarrollo de una nación competitiva, así como con la creciente internacionalización de la educación superior misma. Se puede agregar, en el caso de las universidades públicas, el cumplimiento del deber constitucional de rendición de cuentas. 


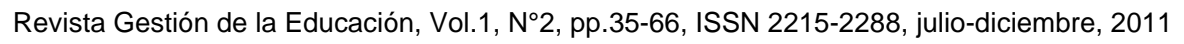

Recientemente, la Conferencia Mundial sobre Educación Superior (CMES), en el año 2009, reforzó la motivación que la UNESCO apuntara desde 1998, al indicar que "la educación superior es un bien público y un imperativo estratégico para todos los niveles de educación. Las bases para la investigación, innovación y creatividad deben ser una materia de responsabilidad y de apoyo económico por los gobiernos como está enfatizado en la Declaración Mundial sobre los Derechos Humanos" (UNESCO, 2009, p.2).

Todas estas razones han sido y continúan siendo motivo para llevar a cabo los procesos de aseguramiento de la calidad, los cuales son parte vital de la gestión universitaria, pues para el desarrollo de una cultura de calidad y para asegurar procesos y productos de excelencia, se requiere de planificación, organización, dirección, evaluación, administración estratégica y liderazgo.

Pires y Lemaitre (2008) comentan que si se pretende garantizar la calidad de una institución o programa, el instrumento más apropiado es el de acreditación, mediante el cual se verifica el cumplimiento de ciertos estándares o criterios públicamente conocidos, puesto que las agencias acreditadoras juzgan los programas o instituciones comparando contra algunos criterios mínimos requeridos para realizar sus funciones, además consideran la capacidad de la carrera o institución de ejecutar planes de mejoramiento.

En Costa Rica, al Sistema Nacional de Acreditación de la Educación Superior (SINAES) pertenecen -en calidad de adherentes- las universidades costarricenses tanto públicas como privadas que hayan cumplido satisfactoriamente los requisitos de adhesión que establece su normativa interna.

La necesidad de evaluar efectos de los procesos de aseguramiento ha sido expresada por personas expertas, quienes indican que "para que los procesos de aseguramiento de la calidad mantengan y actualicen su potencial para la mejora de la educación superior, es esencial que se desarrollen mecanismos e instrumentos para medir su impacto efectivo sobre la calidad de programas e instituciones" (Pires y Lemaitre, 2008, p. 18).

Determinar si una educación es de calidad implica, en última instancia, hacer un juicio en función de determinados criterios y valores. Por tanto, es necesario explicitar un 
Revista Gestión de la Educación, Vol.1, №2, pp.35-66, ISSN 2215-2288, julio-diciembre, 2011

conjunto de conceptos que permitan hacer objetiva su evaluación, mediante dimensiones, criterios e indicadores que deben responder a una estructura conceptual precisa.

Esta investigación se enfocó en dos aspectos de la estructura conceptual necesaria para proponer un modelo: la búsqueda de una conceptualización de calidad en la Educación Superior, acorde con las tendencias internacionales en materia de aseguramiento de la calidad y la búsqueda de un modelo de evaluación de efectos que permita integrar el proceso de acreditación.

De la interacción de los elementos anteriores, se presentan algunos aportes para la creación de un modelo de evaluación de efectos, con el interés de coadyuvar a la evaluación de los efectos de los procesos de acreditación llevados a cabo con el SINAES.

Si se parte de la opinión de personas expertas como Pires y Lemaitre (2008) y Zapata y Tejeda (2009), se considera relevante la evaluación impostergable de los efectos de los procesos de acreditación. De lo anterior, surge la pregunta de investigación:

¿Cómo se pueden evaluar los efectos de los procesos de acreditación con el SINAES en la consecución de la calidad?

Los objetivos de la investigación surgidos de la interrogante son:

\section{Objetivo general}

Brindar aportes para el desarrollo de un modelo de evaluación de los efectos de los procesos de acreditación con SINAES, para una carrera de una universidad estatal costarricense.

\section{Objetivos específicos}

1. Determinar un referente de calidad con sus dimensiones y componentes, con base en las tendencias internacionales en el campo del aseguramiento de la calidad de la educación superior, en particular en el marco de la visión de la UNESCO. 
Revista Gestión de la Educación, Vol.1, №2, pp.35-66, ISSN 2215-2288, julio-diciembre, 2011

2. Determinar la relación de las dimensiones y componentes del Constructo Calidad determinado según el objetivo anterior, con el modelo SINAES y presentar algunos efectos que podrían generarse de los procesos de acreditación como parte de la estrategia del aseguramiento de la calidad.

3. Plantear la conceptualización y algunos requerimientos para el desarrollo de un modelo de evaluación de efectos.

\section{La universidad, calidad de la educación superior}

La preocupación por la calidad en el ambiente universitario es un tema relativamente reciente, así lo afirma Rama (2006), quien ha dividido la evolución de la educación superior en América Latina y el Caribe en tres procesos de reforma, situando la preocupación por la calidad en la última fase, cuya iniciativa surgió en Inglaterra al empezar la década de los años 90, en el siglo XX.

En la última década, la calidad de la educación superior ha sido tema trascendental de debate de los organismos multilaterales y de autores diversos, entre ellos (Abarca, 2002; Albornoz, 2007; Arce y Quirós, 2005; Banco Mundial, 2004; Espinoza y González, 2006; Garbanzo, 2007; Ginés, 2004; Global University Network for Innovation, 2009; L'Ecuyer, 2005; Lemaitre, 2007; López, 2006; Miranda, 2007; OREALC/UNESCO, 2008; Sebastián, 2003; Silva, 2006; Simoneau, 2002; Sol, 2003; Tünnermann, 2000) y otros. Estos indican que su análisis está sustentado en: la masificación; la reducción del financiamiento público; la diversificación de la educación superior; las innovaciones tecnológicas; la exigencia de rendición de cuentas; la Internacionalización; la demanda de formación continua; la interdisciplinariedad; la diversificación de la demanda; la transición hacia economías basadas en el conocimiento; la globalización de los mercados y la competitividad y la demanda creciente de rendición de cuentas (accountability).

La exposición precisa de las motivaciones para evaluar la calidad, contrasta con la escasa concreción de la definición del concepto de la calidad de la educación. La 


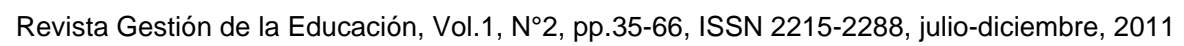

tendencia predominante es hacer aproximaciones al concepto, sin definirlo directamente.

¿Qué se entiende por calidad? Para dar respuesta a esta interrogante hay diversidad de opiniones, lo que se resume en un criterio externado por el Centro Interuniversitario de Desarrollo (CINDA), en el año de 1994. Esta entidad indica que al final de cuentas, calidad es un concepto filosófico. Señala que las definiciones varían y que en cierta forma, reflejan diferentes perspectivas del individuo y la sociedad, por lo que no hay una única y correcta definición de calidad. El término calidad se ve influido por la percepción de quien lo utilice y según las circunstancias en que se emplee.

El concepto de calidad según Cruz (2008) y Dias-Sobrinho (1995), citado por Fernández (2004), es una construcción social, que varía según los intereses de los grupos de dentro y de fuera de las instituciones. Así en una universidad, institución en la cual confluyen intereses de varios grupos, se puede esperar que para los académicos, sea sinónimo de conocimiento; para los empleadores se refiera a las competencias con que los estudiantes egresan y que les permiten integrarse al trabajo; para los estudiantes sería la empleabilidad; la sociedad esperaría ciudadanos respetables y competentes; para el Estado, según la concepción que asuma, puede variar de aspectos vinculados con el desarrollo social y humano a la eficiencia, a los costos y a los requerimientos de capital humano (Cruz, 2008; González y Espinoza, 2008; OREALC/UNESCO, 2008; Suárez y Comboni, 2007).

No se puede definir el concepto de calidad en la educación superior desde un punto de vista único, absoluto y general. La solución práctica a la imposibilidad teórica, ha sido realizar una aproximación basada en criterios de calidad previamente determinados y aceptados, los cuales se utilizan para analizar el quehacer de la universidad.

Dado que según diversos autores (Cruz, 2008; Dias-Sobrinho 2008; González y Ayarza, 2006; OREALC/UNESCO, 2008; Rama, 2008) existen diferentes conceptos y definiciones de calidad en educación superior, cualquier intervención que se realice para asegurarla o incrementarla (Dopico, 2007; Oliva, 2008), implica tener la capacidad para integrar armónica y diferencialmente los distintos componentes involucrados en toda acción educativa, es decir, significa considerar tanto las orientaciones como los procesos y resultados. 
Revista Gestión de la Educación, Vol.1, N², pp.35-66, ISSN 2215-2288, julio-diciembre, 2011

Los elementos anteriormente señalados y el hecho mismo de que existen diversos criterios sobre el término calidad, hacen del aseguramiento de la calidad una labor compleja.

\section{Aseguramiento de la calidad en la educación superior. La acreditación}

El término aseguramiento de la calidad se usa como una "denominación común para una diversidad de mecanismos tendientes a controlar, garantizar y proveer la calidad de las instituciones de educación superior, lo cual contribuye a una mejor comprensión de estos procesos como un aspecto de desarrollo progresivo" (Lemaitre, 2007, p. 6).

Por otra parte, se indica que el "aseguramiento de la calidad se entiende como el cumplimiento de un conjunto de exigencias mínimas que garantizan que las instituciones cuentan con las condiciones requeridas para realizar sus funciones académicas peculiares, esto es, investigación, docencia y extensión" (Dias-Sobrinho, 2006, p. 254). En otras palabras, la calidad de un sistema universitario es el producto de varios factores y procesos, los que son además de abundantes, complejos en sí mismos.

Las instituciones de educación superior han hecho uso de diferentes sistemas o métodos de aseguramiento de la calidad, entre los que se encuentra la acreditación que es el reconocimiento formal y público de que una institución o uno de sus programas o carreras está cumpliendo satisfactoriamente los objetivos declarados y un conjunto de referentes establecidos, criterios o estándares de calidad. Este proceso evaluativo implica el cumplimiento de tres grandes fases: la autoevaluación, la evaluación externa y el seguimiento.

"El supuesto básico de la acreditación institucional, como mecanismo de aseguramiento de la calidad, es la afirmación de que ella promueve un proceso de mejora continua en las instituciones" (Silva, 2006, p.122). 
Revista Gestión de la Educación, Vol.1, №2, pp.35-66, ISSN 2215-2288, julio-diciembre, 2011

\section{La evaluación de calidad y la evaluación de efectos e impactos}

El concepto de evaluación se ha ido transformando desde la revolución industrial, y más especialmente, a comienzos del siglo XX, cuando la teoría administrativa dio cuenta de la importancia de la evaluación para la gerencia de organizaciones.

Siguiendo a Quintero (2007) se puede decir que la evaluación adquiere mayor relevancia debido al carácter cambiante del mundo actual, el cual enfrenta problemas complejos, contingencias e incertidumbres, lo que hace necesario que los procesos de evaluación generen las suficientes señales de alerta para tomar decisiones y poder hacer una buena gestión. Es decir, la importancia de la evaluación consiste en dar información sobre la gestión de los recursos, procesos, productos, efectos e impactos.

La importancia y actualidad de la realización de la evaluación de efectos o impactos, la comenta Oliva (2008):

El desarrollo tecnocientífico permite fundamentar contextualmente, el proceso de evaluación de impacto, puesto que en una sociedad en continuados cambios, la evaluación sigue teniendo el relieve decisivo que la caracteriza, como fuente de retroalimentación de los procesos, mucho más si de la evaluación de impacto se trata, la cual nos inserta directamente en la práctica social y en toda la pertinencia de los procesos de formación. (p. 51)

La misma autora, Oliva (2008) le da gran importancia al método que se utilice para evaluar impactos, en este sentido se trascribe

En toda estrategia investigativa, el método constituye un elemento constitutivo esencial, pues ilumina el proceso mismo de búsqueda de la verdad. No es posible asumir la evaluación en general y la de impacto, en particular, sin la mediación de un método. El modelo elegido es el de "cadena de impactos de GTZ". (p. 58) 
Revista Gestión de la Educación, Vol.1, N², pp.35-66, ISSN 2215-2288, julio-diciembre, 2011

\section{Modelo de cadena de impactos de GTZ}

A continuación, se presentan los principales conceptos necesarios para la comprensión del Modelo de la cadena de impactos de Deutsche Gesellschaft für Technische Zusammenarbeit (GTZ).

Se entiende por proyecto, "un conjunto de medidas delimitado desde el punto de vista funcional, geográfico, económico y temporal, con vistas a alcanzar determinados objetivos" (GTZ, 2008, p. 81).

La "Teoría del programa" (Rossi, 1999, citada por Montero, 2004; Jacoby y Mora, 2008) para la evaluación de programas, es el conjunto de supuestos sobre la forma en que el programa (o proyecto) provoca los beneficios que se espera que produzca, incluyendo las estrategias y tácticas que ha adoptado para lograr sus metas y objetivos. Se divide en "Teoría de los procesos" y "Teoría de los impactos".

La Teoría del proceso describe los planes organizativo y de utilización de servicios del programa. El primero se refiere al personal, sistema de objetivos, recursos financieros, estructura organizacional y de procesos, además de facilidades técnicas; el segundo describe los productos ofrecidos, en cuanto a la ventaja relativa y adecuación a destinatarios.

La Teoría del impacto relata la naturaleza del cambio en las condiciones provocadas por la acción del programa. Esta se refiere a los cambios que ha efectuado el programa en su entorno, sea en los grupos destinatarios, en otros actores, o en los sistemas sociales relevantes. Va más allá de la evaluación de actividades o de productos, o servicios o prestaciones y pone particular énfasis en los efectos directos e indirectos del programa aunque estos estén fuera del control directo de la gerencia del programa. Además, observa cambios a lo largo de toda la cadena de impactos.

Los mismos Jacoby y Mora (2008) indican que la cadena de impactos planteada por la agencia alemana GTZ, es una forma de medir los impactos de un proyecto o programa que involucra: insumos, actividades, producto o resultado, aprovechamiento del producto, efectos e impactos.

Los resultados (output), constituyen la situación o el estado deseado que se 


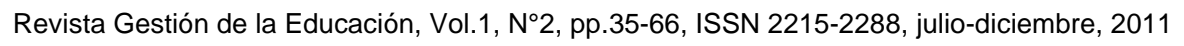

pretende alcanzar con la realización del proyecto. Es decir, los servicios o productos proporcionados por el proyecto, que son de directa responsabilidad de los gestores del proyecto; en esta investigación, equivalen, entre otros, al plan de estudio, proyectos de docencia, investigación y extensión, proyectos de acción social. Hasta aquí llega el límite del ámbito bajo el control de la gestión. Posteriormente, se encuentra el concepto de aprovechamiento del producto o servicio, el cual describe la manera en que los beneficiarios aprovechan el producto o servicio (GTZ, 2007).

Efecto (outcome) es todo comportamiento o acontecimiento del que puede razonablemente decirse que ha sido influido por algún aspecto del programa o proyecto (Bond, 1985, en Cohen y Franco, 1992, citados por Jacoby y Mora, 2008). Los efectos/beneficios generados directamente por el producto o el conjunto de los productos. Define el éxito del proyecto. Los efectos se encuentran fuera de la línea de gestión institucional, es decir, fuera del ámbito de gestión del proyecto, programa o intervención.

El impacto se refiere, según Nebelung (2004), a los efectos de largo plazo, positivos y negativos, primarios y secundarios, producidos directa o indirectamente por las intervenciones del proyecto, sea intencionalmente o no. Un impacto que podría esperarse de las medidas tomadas para la carrera durante el proceso de acreditación, sería por ejemplo un mejoramiento en la calidad de vida en algunos sectores de la sociedad costarricense, que ha logrado una empleabilidad superior a la que hubiera tenido de no haberse realizado el proceso.

La cadena de impactos se presenta como una escala que va subiendo paso por paso hasta llegar a la parte superior (GTZ, 2007). En la parte baja, se comienza con los insumos de un programa y con actividades. Una actividad podría ser por ejemplo la actualización curricular. Varios insumos y actividades tienen como resultado un Output o servicio.

Los outputs son productos o servicios prestados a las personas o grupos a los que está dirigido el programa o el proyecto, por ejemplo, el plan de estudios. Los insumos, actividades y outputs pueden asignarse y controlarse al nivel interno del proyecto.

Una cadena de impacto se refiere a la serie de efectos de cambio generados en los beneficiarios directos. Cada eslabón de la cadena de impacto descansa en una 
Revista Gestión de la Educación, Vol.1, №2, pp.35-66, ISSN 2215-2288, julio-diciembre, 2011

hipótesis de impacto.

La hipótesis tiene dos partes fundamentales. Por un lado, aquellas actividades y resultados que constituyen los servicios o productos proporcionados por el programa a los actores y que se encuentran dentro del ámbito de injerencia permanente, y por otro los beneficios directos $\mathrm{e}$ indirectos percibidos por los actores beneficiarios como consecuencia directa del programa.

Así, estos beneficios se conforman en una cadena de efectos sucesivos y lógicos hasta llegar a cumplir los objetivos del programa. Los cambios provocados por el uso de los servicios o productos son definidos como efectos o beneficios directos. Este nivel marca el final de la influencia directa de un proyecto. Según GTZ (2007), los cambios más allá de este nivel no se pueden atribuir directamente a un proyecto, ya que están, por definición, más allá de su control directo.

Se maneja el concepto de brecha de atribución cuando en la línea de beneficios es difícil poder atribuir el cambio solamente a los resultados del programa (GTZ, 2007). Es claro que las acciones de otros proyectos y actores, contribuyen también a lograr beneficios superiores y de impacto para una región, grupo social o sector económico.

Metodológicamente, la brecha de atribución, como un elemento esencial de la cadena de impactos, marca este punto y aclara la diferencia entre efectos directos y los llamados Beneficios indirectos o impactos. Los beneficios indirectos no se pueden atribuir por completo al trabajo del programa o proyecto debido a una gran cantidad de factores externos que influyen en este nivel.

Los niveles de actividades y de servicios o productos, es decir, el ámbito de gestión de un proyecto se encuentra en el área de monitoreo, mientras que la medición del resto de niveles se realiza a través de evaluaciones de impacto.

\section{Metodología}

El enfoque de investigación que se aplica en el estudio es el cualitativo, por tanto es descriptivo y explicativo. Específicamente, para el desarrollo de la propuesta del 
Revista Gestión de la Educación, Vol.1, №2, pp.35-66, ISSN 2215-2288, julio-diciembre, 2011

modelo, se hace uso de un enfoque mixto, con elementos tanto del enfoque cualitativo como del cuantitativo.

Al ser la evaluación de efectos un tema novedoso fue necesario hacer un recorrido exploratorio por diferentes enfoques, que en general apuntaban a la necesidad de encontrar un referente contra el cual comparar.

La investigación abordó la búsqueda de un referente que conceptualizara la calidad como un enfoque multidimensional, un constructo de calidad y la búsqueda de un modelo que respondiera a los requerimientos de la evaluación de efectos de los procesos de acreditación.

Para el constructo, se eligió el concepto de calidad de la educación dado por la OREALC/UNESCO a finales del 2008, conformado por cinco dimensiones esenciales a saber; relevancia, pertinencia, equidad, eficiencia y eficacia, al cual se le asociaron algunos componentes. Para sustentar esta propuesta se amplió el análisis con criterios de expertos en el tema de acreditación, lo cual permitió conocer las diferentes dimensiones de la vivencia de las carreras que siguen un proceso de acreditación, además se discutió a partir de sus experiencias- sobre el concepto de calidad. El instrumento utilizado fue la entrevista abierta.

Paralelamente, se realizó la búsqueda de un modelo de evaluación de efectos, lo cual conduce a la elección de la teoría de la Cadena de Impactos de GTZ, la Agencia Alemana de Cooperación. Esta fue concebida, según GTZ (2007), precisamente para medir efectos e impactos. Utilizando como instrumento la entrevista abierta a los docentes en la teoría de la Cadena de Impactos, se discutió sobre la posibilidad de adecuar la propuesta a un nuevo objeto y de realizar una evaluación de efectos de la acreditación, considerando a la acreditación como una intervención en la carrera.

Además, se realizó un análisis paralelo, con el fin de que los informantes que se constituyeron en fuentes primarias de información, cooperaran al revisar la inclusión de conceptos provenientes de las fuentes secundarias.

Para el diseño de la propuesta, se tomó como punto de partida que el interés está centrado en los efectos relacionados con el aseguramiento de la calidad, desde la 


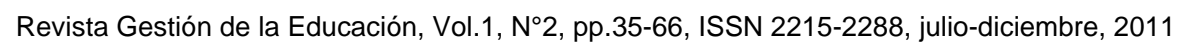

perspectiva del constructo determinado de antemano. En el momento de la evaluación, el efecto esperado se convierte en la variable a analizar.

\section{Resultados}

\section{La Teoría del programa: punto de unión para la evaluación de efectos}

La premisa básica desde la cual se desarrolla el diseño de evaluación de los efectos, donde el término "efecto" se entiende como todo comportamiento o acontecimiento del que puede razonablemente decirse que ha sido influido por algún aspecto de la acreditación, es que, la llamada "Teoría de los procesos" la cual forma parte de la "Teoría del programa", según terminología de la Cadena de impactos de GTZ, está desarrollada en el Modelo de acreditación del SINAES. Por su parte, la "Teoría de los impactos" se deriva de relaciones causa efecto. La siguiente figura expone esta relación:

Figura 1

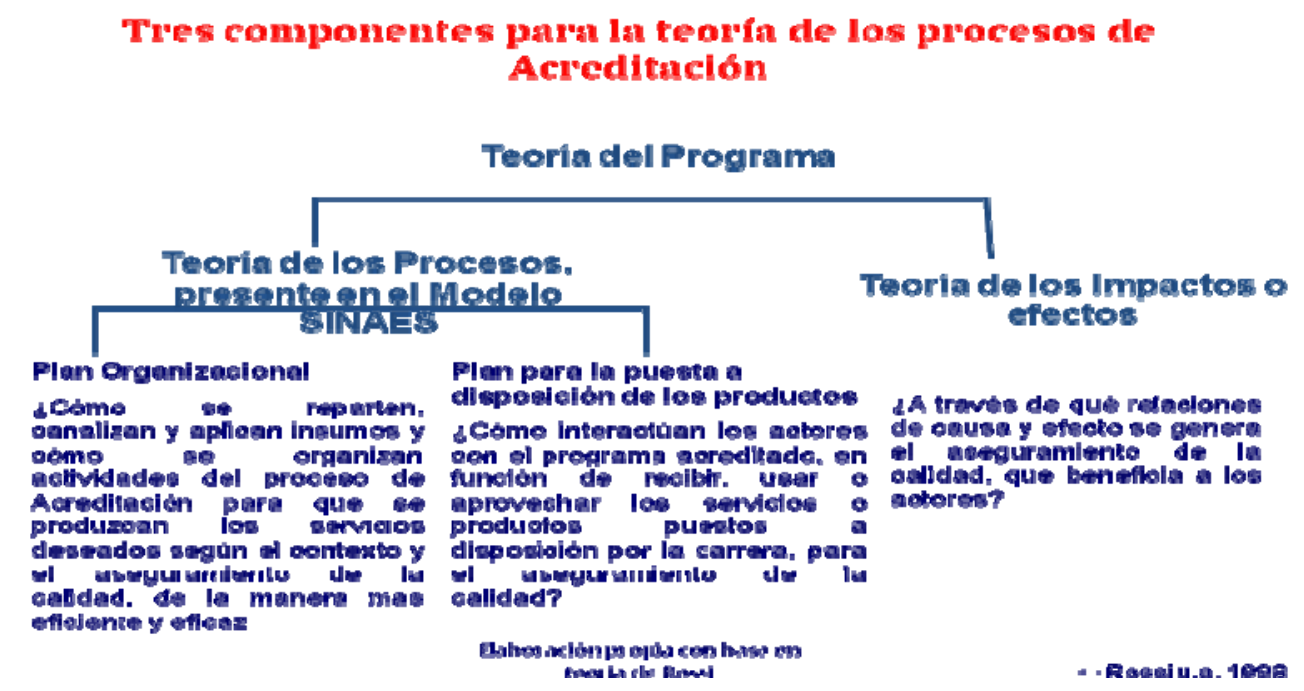

Fuente: Elaboración propia con base en teoría de Rossi, citado por Montero (2004); Jacoby y Mora (2008). 
Revista Gestión de la Educación, Vol.1, N², pp.35-66, ISSN 2215-2288, julio-diciembre, 2011

Es decir, en los criterios de evaluación del modelo de acreditación se encuentran perfiladas las principales decisiones que debe tomar la carrera para acreditarse y para asegurar la calidad, como se observa en la siguiente tabla:

Tabla No 1

Niveles de decisión relacionados con las cuatro dimensiones del Modelo SINAES

\begin{tabular}{|l|l|}
\hline Dimensiones modelo SINAES & Niveles de decisión \\
\hline Contexto & Decisiones de adecuación al contexto \\
\hline Insumos & Decisiones de planeación \\
\hline Procesos & Decisiones de implementación \\
\hline Productos & Decisiones de continuidad o relevamiento \\
\hline
\end{tabular}

Fuente: Elaboración propia con base en teoría del CIPP, SINAES, 2009.

Una vez expuestos estos antecedentes básicos, se presentan los resultados obtenidos al desarrollar los objetivos de la investigación.

\section{Constructo Calidad}

En razón de que UNESCO es la agencia de Naciones Unidas especializada en la educación, que entre sus prioridades se encuentra la Educación Superior y es una autoridad legitimada a nivel mundial, se eligió en esta investigación la estructura conceptual de la calidad expuesta por OREALC/UNESCO (2008), desde una visión de promoción de los derechos humanos, de la cual la educación superior se considera como un bien público, enfoque coincidente con la Declaración de los Derechos Humanos y con la posición asumida por la Conferencia Mundial de la Educación Superior, celebrada en julio de 2009.

En este sentido, la educación se concibe como un medio para que el ser humano se desarrolle plenamente como tal, ya que gracias a ella crece y se fortalece como persona y como especie que contribuye al desarrollo de la sociedad, transmitiendo y compartiendo sus valores y su cultura. 
Revista Gestión de la Educación, Vol.1, №2, pp.35-66, ISSN 2215-2288, julio-diciembre, 2011

Sobre esta base la OREALC/UNESCO (2008) plantea un concepto de calidad de la educación conformado por cinco dimensiones esenciales y estrechamente imbricadas, al punto que la ausencia de una implicaría una concepción equivocada de la calidad de la educación dentro de un enfoque de derechos humanos, como se muestra en la siguiente figura.

Figura 2

Concepto de calidad

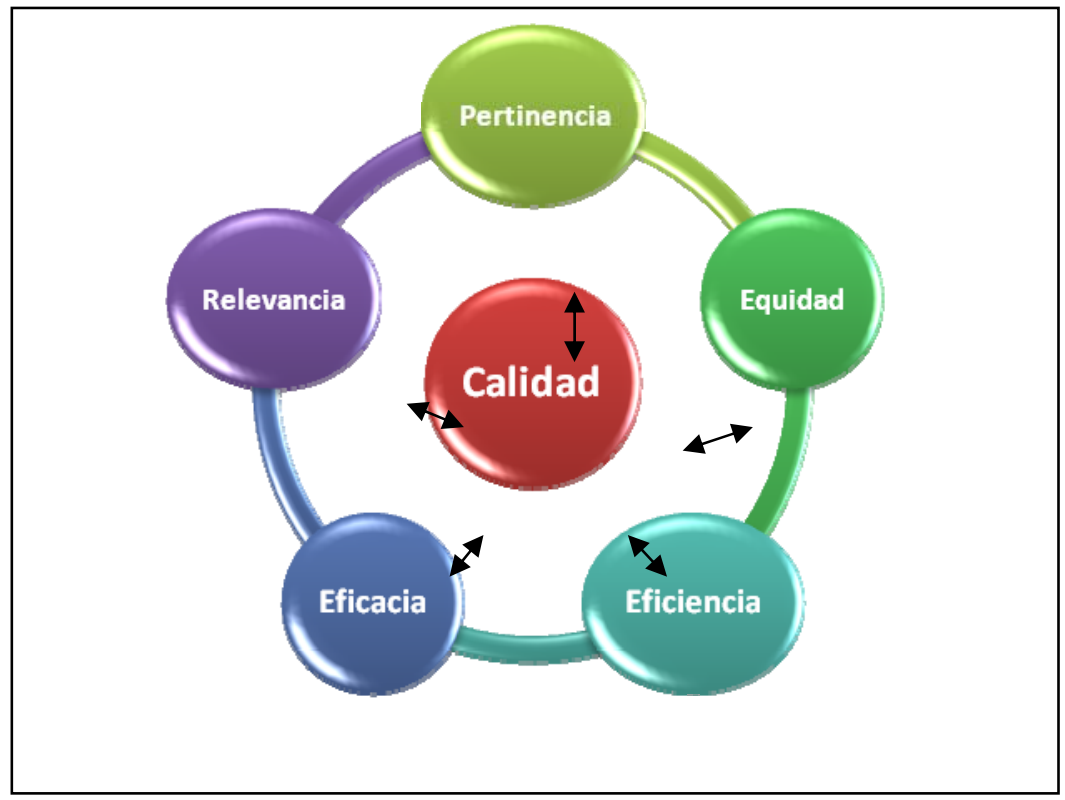

Fuente: Elaboración propia con base en OREALC/UNESCO, 2008.

Para efectos de la investigación, algunas dimensiones del constructo de calidad se desagregaron en componentes, los cuales se determinaron a partir de la explicación amplia de la definición de las dimensiones dada por OREALC/UNESCO (2008) y otros autores. Así, las dimensiones y componentes del constructo calidad determinado en esta investigación son: 
Revista Gestión de la Educación, Vol.1, №2, pp.35-66, ISSN 2215-2288, julio-diciembre, 2011

Relevancia comprende:

- Compromiso para lograr la conformidad con el estado del arte de la disciplina, lo que significa que el plan de estudios contempla los avances de la comunidad académica internacional en los distintos campos de la ciencia, la cultura y el conocimiento.

- Compromiso con el desarrollo humano integral y sostenible, lo que se refiere a que el plan de estudios provee una formación plena que ayuda al estudiante a estructurar su identidad y a desarrollar sus capacidades para participar en la construcción de la sociedad, como proceso permanente que facilita el aprendizaje, el desarrollo de competencias, la experiencia y la incorporación plena de los valores, afectos y de sus potencialidades, tanto individuales como sociales y fomenta la participación en propuestas de cambio para lograr un mundo más justo y más equitativo (Martínez, 2006).

- Desarrollo de la investigación y extensión, es decir, se relaciona con la promoción y ejecución de proyectos de investigación, acción social y extensión. La investigación como forma de preparar a los docentes para una enseñanza de calidad, que involucra en alguna medida a los estudiantes. La acción social y la extensión, ligados a la sociedad y sus desafíos.

\section{Equidad comprende:}

- Igualdad de oportunidades en el acceso y permanencia, es decir, la equidad es sensible a las diferencias entre los seres humanos por lo que, para garantizar igualdad de oportunidades, es necesario apoyar con mayores recursos a los grupos más vulnerables. El pleno ejercicio del derecho a una educación de calidad para todos exige garantizar el derecho a la igualdad de oportunidades, es decir, proporcionar más a quien así lo necesita y dar a cada uno las ayudas y recursos que requiere para estar en igualdad de condiciones de aprovechar las oportunidades educativas (Blanco, 2006; CRES, 2008; Sen, 1999, citados por Arrieta, 2009). 
Revista Gestión de la Educación, Vol.1, №2, pp.35-66, ISSN 2215-2288, julio-diciembre, 2011

- Igualdad de oportunidades en la apropiación del conocimiento que se refiere al deber de los sistemas educativos de que todos los alumnos desarrollen las mismas competencias que les permitan la participación y actuación en la sociedad, y el desarrollo de proyectos de vida; pero también se ha de ofrecer la oportunidad de incorporar otros aprendizajes que sean relevantes en función de los diferentes contextos y culturas, y de los múltiples talentos, intereses y motivaciones de cada persona (López, 2005).

- Estrategia educativa que integra diversidad y derechos, la cual se relaciona con que la Universidad ayuda a comprender, interpretar, preservar, reforzar, fomentar y difundir las culturas nacionales y regionales, internacionales e históricas, en un contexto de pluralismo cultural y de diversidad cultural. La educación superior forma a los estudiantes para que participen activamente en la sociedad, para que defiendan los derechos humanos, la democracia, el desarrollo sostenible, la cultura de paz, la tolerancia y la equidad, entre otros valores esenciales (López, 2005).

\section{Pertinencia comprende:}

- Flexibilidad del currículo y una estructura de este que define un núcleo o corpus básico y un componente flexible. El primero de ellos contiene las unidades de organización curricular (asignaturas, módulos, núcleos, etc.,) las cuales deben cursar todos los estudiantes matriculados en un programa de pregrado y que son básicas e indispensables. El segundo elemento de la estructura curricular es el denominado componente flexible que contiene las unidades de organización curricular que elige el estudiante de acuerdo con sus necesidades, intereses, problemas y aptitudes.

- Formación de los docentes, que refiere a la selección esmerada del personal y su perfeccionamiento constante, en particular mediante la promoción de planes de estudio adecuados para el perfeccionamiento del personal universitario, incluida la metodología del proceso pedagógico (UNESCO, 1998). 
Revista Gestión de la Educación, Vol.1, N², pp.35-66, ISSN 2215-2288, julio-diciembre, 2011

- Formación de los administrativos, es decir, selección esmerada del personal y su perfeccionamiento constante.

- Internacionalización que se relaciona con el intercambio de conocimientos, la creación de sistemas interactivos, la movilidad de profesores y estudiantes y los proyectos de investigación internacionales.

\section{Análisis del modelo del SINAES para la acreditación oficial de carreras de grado (2008), a partir del constructo de calidad determinado}

Con base en la propuesta de constructo de calidad definido, se analizó la relación con los criterios del modelo propuesto por el SINAES. Solo se exceptúan de esa relación detallada los criterios que corresponden a admisibilidad y sostenibilidad, los primeros por ser parte de un paso previo al inicio del proceso de acreditación y el segundo porque se parte del supuesto de que la evaluación de efectos e impactos solo podría realizarse en un contexto de sostenibilidad del proceso de acreditación.

Previamente a realizar el análisis pretendido sobre los efectos, se reformularon los criterios de las dimensiones del modelo SINAES, es decir, del Manual de Acreditación Oficial de Carreras de Grado del Sistema Nacional de Acreditación de la Educación Superior (SINAES, 2009), de modo que en su redacción estuvieran implícitos la dimensión y el componente del cual forman parte, es decir, las dimensiones Relación con el contexto, Recursos, Proceso Educativo y Resultados. Los respectivos componentes, se incluyeron en sus correspondientes criterios. A estos criterios se les llamó, para efectos de identificación, "criterios compendiados".

A partir de lo indicado, el análisis consistió en relacionar los criterios compendiados, que constituyen el conjunto de condiciones que la carrera debe cumplir como normas o patrones para acreditarse, con las dimensiones y componentes propuestos como parte del Constructo Calidad. Es decir, se clasifica cada criterio compendiado, según su cumplimiento contribuya con la búsqueda y consecución de la 
Revista Gestión de la Educación, Vol.1, №2, pp.35-66, ISSN 2215-2288, julio-diciembre, 2011

pertinencia, relevancia, equidad, eficiencia o eficacia, dimensiones del Constructo Calidad.

Al poner a contraluz el modelo del SINAES con el constructo calidad, resulta validado como un modelo en armonía con las tendencias actuales de calidad de la Educación Superior.

Se muestra en la siguiente tabla un ejemplo de la interrelación de las dimensiones y componentes determinados en el constructo Calidad, con los criterios compendiados que corresponden a los criterios 1.1 .1 y 1.1.2, del componente "Información y promoción" de la dimensión "Relación con el contexto", del modelo SINAES:

Tabla No 2

Ejemplo de la interrelación del modelo del SINAES con las dimensiones y los componentes que caracterizan la calidad según el constructo determinado

\begin{tabular}{|l|l|l|}
\hline \multicolumn{1}{|c|}{ Criterio compendiado } & \multicolumn{1}{|c|}{$\begin{array}{c}\text { Dimensión } \\
\text { según } \\
\text { Constructo } \\
\text { Calidad }\end{array}$} & $\begin{array}{c}\text { Componente de la } \\
\text { dimensión }\end{array}$ \\
\hline $\begin{array}{l}1.1 .1 \text { La carrera debe brindar información de } \\
\text { los trámites de ingreso, duración, convalidaciones y } \\
\text { reconocimientos y las tarifas de los trámites } \\
\text { académico-administrativos, por medios que permitan } \\
\text { acceso público. }\end{array}$ & Equidad. & $\begin{array}{l}\text { Igualdad de } \\
\text { oportunidades en el } \\
\text { acceso y permanencia. }\end{array}$ \\
\hline $\begin{array}{l}1.1 .2 \text { La carrera debe informar al estudiante en } \\
\text { forma oportuna y veraz, sobre el plan de estudio, el } \\
\text { tempo promedio de graduación, los costos, la } \\
\text { normativa, fechas, trámites y servicios. }\end{array}$ & Equidad. & $\begin{array}{l}\text { Igualdad de } \\
\text { oportunidades en el } \\
\text { acceso y permanencia. }\end{array}$ \\
\hline
\end{tabular}

Fuente: Elaboración propia con base en SINAES, 2009 y el Constructo Calidad de este estudio.

\section{Hacia un modelo para evaluar los efectos de los procesos de acreditación de carreras en las universidades estatales de Costa Rica: propuesta}

La investigación propone algunos elementos para construir un modelo de evaluación de los efectos, con el fin de conocer en qué medida el proceso de acreditación, 
Revista Gestión de la Educación, Vol.1, №2, pp.35-66, ISSN 2215-2288, julio-diciembre, 2011

coadyuva a que la universidad pública costarricense cumpla su papel dentro de la sociedad, en el marco de las tendencias mundiales en Educación Superior.

Se entiende el proceso de acreditación como una sucesión de fases que constituyen un programa o proyecto, según la terminología de la Cadena de Impactos de la GTZ. Lo anterior por tanto estas fases se ordenan según un conjunto articulado de actividades, orientado hacia un objetivo: el aseguramiento de la calidad, mediante una metodología definida, con recursos definidos (personal, presupuesto) y con una duración limitada.

Además este programa, el proceso de acreditación, es una intervención en el accionar de la carrera, para afianzar o modificar, tanto la gestión académica como la administrativa, con el fin de lograr el aseguramiento de la calidad.

De la unión entre ambos, gestión de la carrera y el proceso de acreditación, surgirán los efectos deseados en el proceso de aseguramiento de la calidad. Asimilada esta última al Constructo Calidad, con las dimensiones de Relevancia, Pertinencia, Eficiencia, Equidad y Eficacia.

Como punto de partida para el desarrollo de un Modelo de evaluación de efectos y dentro de la metodología de la Cadena de impactos de la GTZ, la llamada "Teoría del Programa", debe estar enlazada y estrechamente imbricada desde que se toma la decisión de iniciar un proceso de acreditación, es decir, las decisiones de planificación y organización de las actividades de una carrera, así como la forma en que se dan las interacciones con todos los actores involucrados en el fenómeno educativo, debe prever la evaluación de los efectos directos e impactos de ese accionar de la carrera.

Lo anterior, implicaría que desde un inicio se constituya una cadena de impacto, la cual se entendería como la serie de efectos de cambio generados en los actores o beneficiarios directos de la acreditación, como parte del proceso de Aseguramiento de la calidad de una carrera. En otras palabras, las acciones tomadas por la carrera para lograr el aseguramiento de la calidad, en el contexto de la acreditación, vendrían a constituirse en las causas que provocarían efectos en los actores, entre estos últimos: estudiantes, graduados, empleadores y la sociedad. Esta relación se ilustra de seguido: 
Revista Gestión de la Educación, Vol.1, №2, pp.35-66, ISSN 2215-2288, julio-diciembre, 2011

Figura 3

Lógica de la relación entre el criterio compendiado, el constructo calidad y la variable-efecto

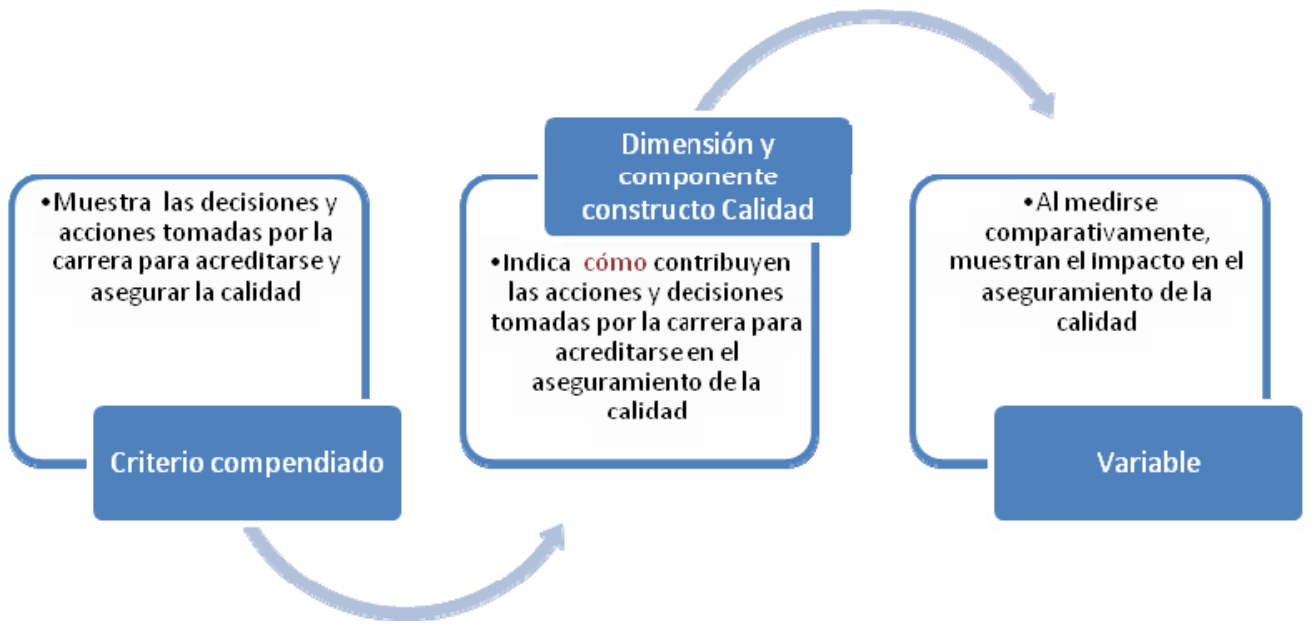

Fuente: Elaboración propia.

Esta relación daría lugar a lo que en terminología GTZ se denomina "Modelo causal para el impacto de los programas", de la siguiente manera:

Figura 4

Interrelación en la teoría de los programas, según terminología GTZ, del Modelo SINAES y el modelo propuesto

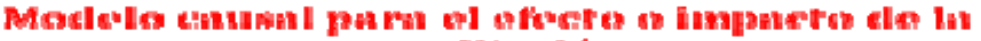
arcicesiltarelobr

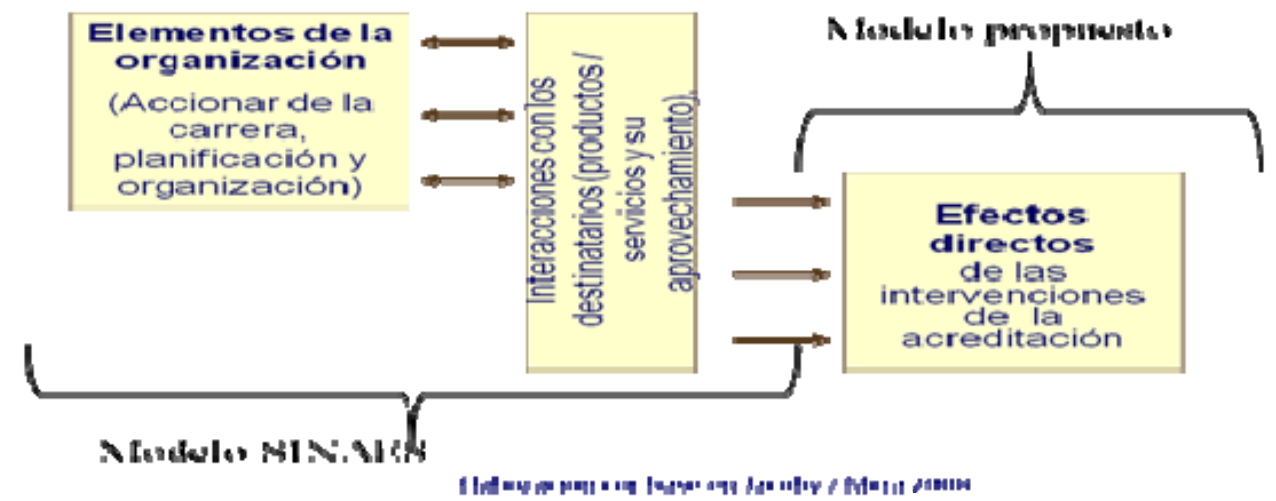

Fuente: Elaboración propia con base en Jacoby (2008). 
Revista Gestión de la Educación, Vol.1, №2, pp.35-66, ISSN 2215-2288, julio-diciembre, 2011

La acreditación interviene en la carrera tanto en lo relativo a la administración como a la academia, involucra todos los elementos de la organización de la carrera. El proceso de acreditación conduce a revisar y de ser necesario, modificar la distribución de insumos y la forma de llevar a cabo los procesos para que los bienes y servicios cumplan con los objetivos del aseguramiento de la calidad.

Como ejemplo, se expone el funcionamiento del modelo para la variable empleabilidad de los egresados, en relación con el criterio compendiado 1.3.1, que muestra la relevancia de la carrera en el contexto.

Cada eslabón de la cadena de impacto descansa en una hipótesis de causa y efecto. La hipótesis tiene dos partes fundamentales: por un lado aquellas actividades y resultados que constituyen los servicios proporcionados a los beneficiarios (teoría de los procesos) y que se encuentran dentro del ámbito de injerencia permanente del programa, es decir, el ámbito de la carrera; y por otro los beneficios directos e indirectos percibidos por los actores y beneficiarios como consecuencia directa de la utilización de los servicios o productos (teoría de los impactos).

Para la variable empleabilidad de los egresados, en relación con el criterio compendiado 1.3.1, se presentan los supuestos o hipótesis necesarios para que se efectúe la relación causa-efecto.

\section{Tabla 3}

Cadena de causa y efecto necesaria para que se de la cadena de impactos y efectos para la variable empleabilidad

\begin{tabular}{|l|l|}
\hline Enunciado & $\begin{array}{l}\text { Lugar que ocupa en la cadena de efectos o } \\
\text { impactos }\end{array}$ \\
\hline $\begin{array}{l}\text { El plan de estudio refleja el estado del } \\
\text { arte de la disciplina y la realidad del } \\
\text { contexto nacional y del mercado laboral }\end{array}$ & Producto \\
\hline $\begin{array}{l}\text { Si el plan de estudios comprende los } \\
\text { avances de la comunidad académica } \\
\text { internacional en los distintos campos de } \\
\text { la ciencia, la cultura y el conocimiento, se } \\
\text { influye positivamente en la calidad, } \\
\text { específicamente en la relevancia. }\end{array}$ & Hipótesis de causa y efecto \\
\hline $\begin{array}{l}\text { Los egresados han adquirido capacidad y } \\
\text { desarrollado nuevas competencias }\end{array}$ & Aprovechamiento del producto \\
\hline Número publicado el 20 de diciembre de 2011 URL: http://revistadigital.eae.fcs.ucr.ac.crl \\
\hline
\end{tabular}


Revista Gestión de la Educación, Vol.1, №2, pp.35-66, ISSN 2215-2288, julio-diciembre, 2011

\begin{tabular}{|l|l|}
\hline $\begin{array}{l}\text { El desempeño de los graduados es } \\
\text { percibido como superior al promedio. }\end{array}$ & Hipótesis causa y efecto \\
\hline $\begin{array}{l}\text { Los egresados del programa acreditado } \\
\text { han incrementado su empleabilidad }\end{array}$ & Efecto \\
\hline Aumento de ingresos & Efecto indirecto \\
\hline
\end{tabular}

Fuente: Elaboración propia.

La relación lógica se muestra en la siguiente figura. Las flechas simbolizan las acciones emprendidas por la carrera para cumplir con el criterio 1.3.1. del Modelo SINAES, es decir, las medidas que se tomaron para asegurar la calidad, de forma que el producto que entrega, plan de estudio, sea relevante.

Figura 5

Lógica de la relación entre el criterio compendiado 1.3.1, el constructo calidad y la variable efecto empleabilidad

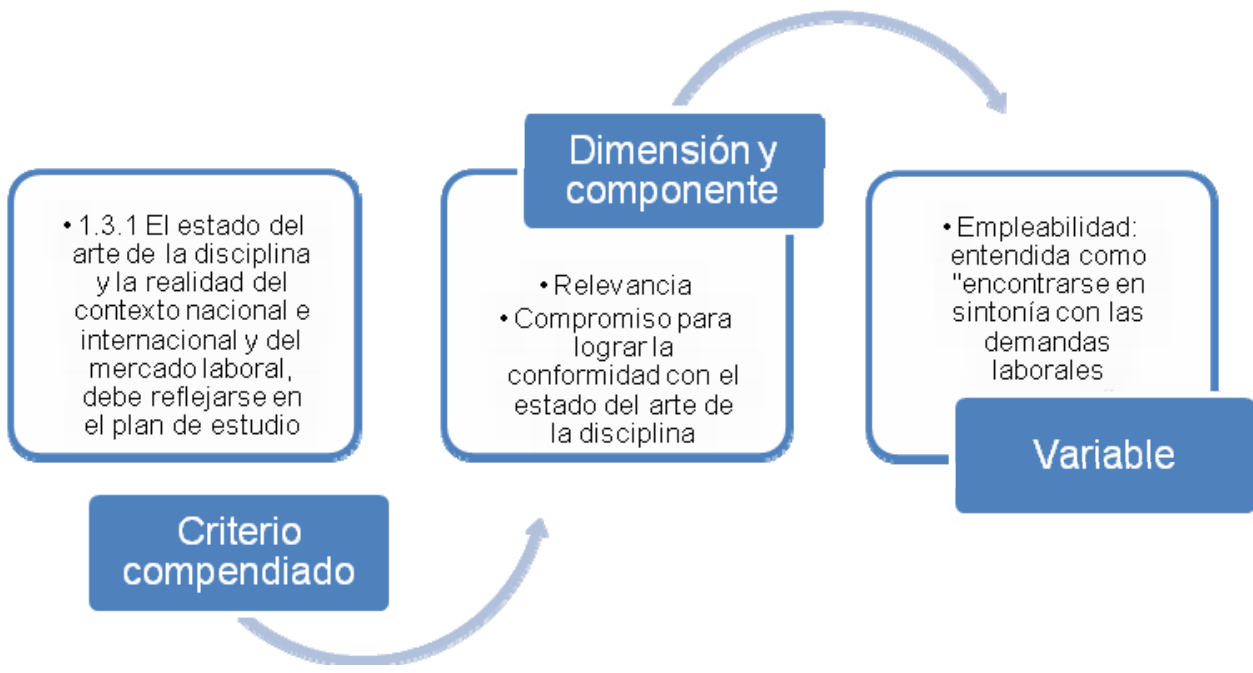

Fuente: Elaboración propia.

En la siguiente tabla se muestran los posibles efectos de las acciones emprendidas por la carrera en el aseguramiento de la calidad, específicamente la 


\section{GeGestión:}

Revista Gestión de la Educación, Vol.1, N², pp.35-66, ISSN 2215-2288, julio-diciembre, 2011

conformidad con el estado del arte de la disciplina y una vez aprovechado por los actores beneficiarios:

\section{Tabla 4}

Determinación de los posibles efectos de las acciones tomadas por la carrera en atención a los criterios del SINAES, específicamente para el criterio compendiado 1.3.1

\begin{tabular}{|c|c|c|c|}
\hline $\begin{array}{l}\text { Criterio compendiado } \\
\text { (resume el criterio, el } \\
\text { componente y la } \\
\text { dimensión del } \\
\text { SINAES) } \\
\end{array}$ & $\begin{array}{l}\text { Dimensión a la } \\
\text { dentro del } \\
\text { Constructo } \\
\text { Calidad }\end{array}$ & $\begin{array}{l}\text { Componente de la } \\
\text { dimensión del } \\
\text { Constructo Calidad }\end{array}$ & $\begin{array}{l}\text { Posibles efectos de las acciones } \\
\text { tomadas durante el proceso de } \\
\text { acreditación; los que se } \\
\text { constituyen en variables de } \\
\text { análisis para fines de evaluación }\end{array}$ \\
\hline $\begin{array}{l}\text { 1.3.1 El estado del arte } \\
\text { de la disciplina y la } \\
\text { realidad del contexto } \\
\text { nacional e internacional } \\
\text { y del mercado laboral } \\
\text { debe reflejarse en el } \\
\text { plan de estudio. }\end{array}$ & Relevancia & $\begin{array}{l}\text { Compromiso para } \\
\text { lograr la conformidad } \\
\text { con el estado del } \\
\text { arte de la disciplina }\end{array}$ & $\begin{array}{l}\text { Graduados: } \\
\text { - Incremento empleabilidad } \\
\text { Estudiantes: } \\
\text { - Incremento en posibilidad de } \\
\text { movilidad. } \\
\text { Empleadores: } \\
\text { - Mayor disponibilidad de } \\
\text { contratación de graduados por los } \\
\text { conocimientos de estos. } \\
\text { - Incremento de disponibilidad de } \\
\text { personal calificado. } \\
\text { - Satisfacción con el desempeño de } \\
\text { los egresados como personal en } \\
\text { la empresa } \\
\text { Sociedad: } \\
\text { - Mayor posibilidad de contar con } \\
\text { recurso humano calificado para la } \\
\text { resolución de problemas } \\
\text { nacionales y con capacidad de } \\
\text { innovar. }\end{array}$ \\
\hline
\end{tabular}

Fuente: Elaboración propia con base en diferentes autores (Blanco, 2006; CRES, 2008; Sen, 1999, citados por Arrieta, 2009; Dias-Sobrinho, 2008; GUNI, 2007; López, 2006; Martínez, 2006; UNESCO, 2009).

La cadena de impactos para la variable empleabilidad de los graduados, en relación con las acciones seguidas por la carrera para cumplir con el criterio compendiado 1.3.1 y asegurar la relevancia en cuanto el Plan de estudios refleje el estado del arte, se presenta en la siguiente figura. En esta se representa la relación entre insumos, proceso, producto y efecto, y las varias hipótesis de causa y efecto. 
Figura 6

Cadena de impactos variable empleabilidad

\section{Relevancia: Compromiso para lograr la conformidad con el estado del arte de la disciplina}

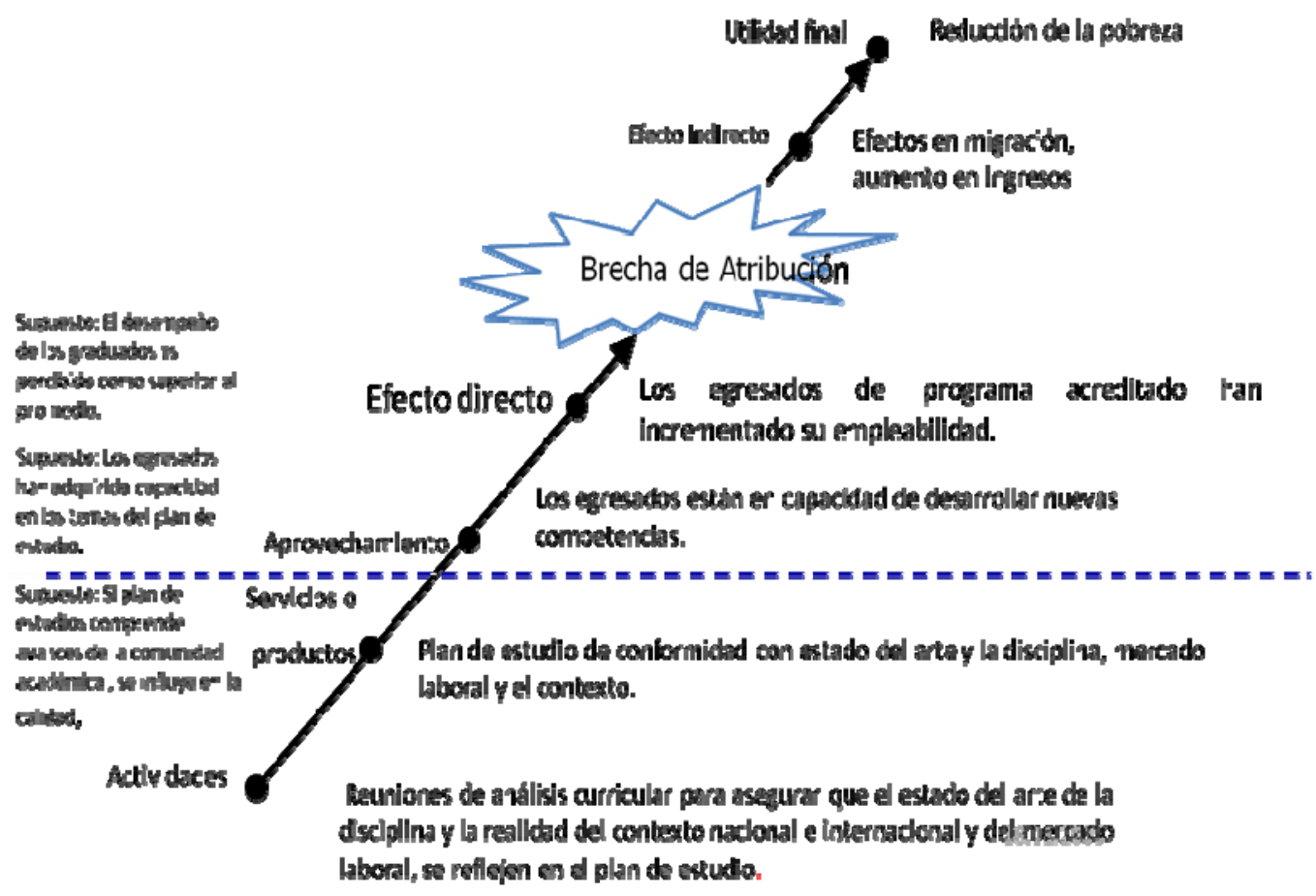

Fuente: Elaboración propia con base en Jacoby (2008).

Para la variable empleabilidad de los graduados, los indicadores propuestos en la investigación son los siguientes: inserción laboral; inserción laboral en el campo de estudio; plazo trascurrido para inserción después graduación, satisfacción laboral; y valoración de la calidad del trabajo según necesidades y expectativas.

El significado de cada indicador es el siguiente:

- Inserción laboral: si el graduado tiene o no, cualquier tipo de trabajo remunerado, incluido el ser emprendedor. 
Revista Gestión de la Educación, Vol.1, №2, pp.35-66, ISSN 2215-2288, julio-diciembre, 2011

- Inserción laboral en el campo de estudio: si el graduado trabaja o no, en su campo de estudio.

- Plazo trascurrido para inserción después de graduación o cambio de trabajo: número de meses transcurridos desde la graduación para insertarse en el mundo laboral.

- Satisfacción laboral: opinión del graduado sobre las labores que desempeña.

- Valoración de la calidad del trabajo: opinión del graduado que trabaja en relación con características de su trabajo como, ingresos, estabilidad, posibilidad de desarrollo en comparación con sus necesidades y expectativas.

Las variables se medirían para la primera cohorte de graduados con el plan de estudios acreditado. En la investigación se determinaron las categorías para los indicadores elegidos. Los momentos de medición serían, dos años después desde la cohorte primera de graduados, y cuatro años después, con el fin de que los empleadores se hayan formado una opinión.

\section{Conclusiones de la investigación}

Las principales contribuciones realizadas con la investigación son:

- Se conceptualiza la idea de efecto de la acreditación en el aseguramiento de la calidad desde un enfoque novedoso, porque se propone evaluar los efectos fuera de la línea de gestión institucional. Esto se considera un aporte relevante porque en la revisión teórica efectuada se pudo observar que las propuestas existentes se refieren más bien a la evaluación de productos o resultados -según la terminología GTZ- y no de efectos, es decir, se circunscriben al ámbito de la carrera o a lo sumo de la universidad. Un efecto, en cambio, siempre dentro del marco analítico elegido, supone un cambio que trasciende el ámbito de la institución. 
Revista Gestión de la Educación, Vol.1, №2, pp.35-66, ISSN 2215-2288, julio-diciembre, 2011

- La integración entre el Modelo del SINAES y la teoría de GTZ, mostrando la relación causa-efecto, entre el cumplimiento de criterios de calidad y los efectos que podrían esperarse de dicha conformidad, posibilita para que una carrera pueda realizar la evaluación de efectos, aunque esta no haya sido planeada de antemano.

- El constructo calidad elegido hace patente que la educación superior que responde a esos parámetros es efectivamente un poderoso agente de cambio del ser humano y de la sociedad.

Los principales retos para continuar con el análisis de la evaluación de efectos de los procesos de aseguramiento de la calidad son:

- Enriquecer y reajustar el modelo teórico con la participación de un equipo interdisciplinario. El objetivo de este trabajo ha sido generar un primer aporte que aclare la relevancia del tema e ilumine posibles caminos para su abordaje.

- También se requiere crear conciencia entre los actores de la educación superior acerca de la importancia de la evaluación de efectos. Este sería un paso fundamental para lograr su respaldo para la integración del modelo propuesto con el de acreditación.

\section{Referencias bibliográficas}

Abarca, A. (2002). Financiamiento y nuevas formas de gerencialismo universitario. Revista Educación 26(1), 73-82. Recuperado de http:// www.redalyc.uaemex.mx/redalyc/pdf/440/44026107.pdf

Albornoz, M. (2007). ¿Calidad Educativa significa lo mismo para todos los actores escolares? Mayéutica Educativa. Recuperado de http://mayeuticaeducativa.idoneos.com/index.php/347332

Arce D., R. y Quirós V., M. (2005). Acreditación internacional: el caso de la agencia de acreditación canadiense CCPE-CEAB en las universidades costarricenses. Revista 
Revista Gestión de la Educación, Vol.1, №2, pp.35-66, ISSN 2215-2288, julio-diciembre, 2011

Actualidades Investigativas en Educación, 5 (número especial), 1-19. Recuperado de http://revista.inie.ucr.ac.cr/articulos/extra-cea/agencia.php

Arrieta, R. (2009). La teoría de la justicia de Amartya Sen. Blog luscontifil. Recuperado de http://iusconstifil.blogspot.com/2009/02/la-teoria-de-la-justicia-de-amartya-sen.html

Banco Mundial (2002). Constructing Knowledge Societies: New Challenges for Tertiary Education. Banco Mundial, Washington, D.C.

Centro Interuniversitario de Desarrollo [CID]. (1994). Manual autoevaluación para instituciones de Educación Superior. Santiago, Chile: CINDA/PROMESUP/OEA.

Cruz, Y. (2008). La acreditación como mecanismo para la garantía del compromiso social de las universidades. Tesis presentada en opción al grado científico de Doctor en Ciencias de la Educación Universitat Politécnica de Catalunya, España. Recuperado de http://www.tesisenxarxa.net/TESIS UPC/AVAILABLE/TDX0602109-095706//01Ycl01de01.pdf

Deutsche Gesellschaft für Technische Zusammenarbeit [GTZ]. (2008). El mundo de conceptos de la GTZ. Recuperado de http://www.gtz.de/sp/publikationen/begriffswelt-gtz/en/9071.htm

Deutsche Gesellschaft für Technische Zusammenarbeit [GTZ]. (2008). Guía de Monitoreo de Impacto. Recuperado de www.gtz.de/en/dokumente/sp-sl-guia-monitoreo-deimpacto.pdf+cadena+de+impacto+gtz\&hl=

Dias-Sobrinho, J. (2008). Calidad, pertinencia y responsabilidad social de la universidad latinoamericana y caribeña. En: Tendencias de la Educación Superior en América $\begin{array}{lllll}\text { Latina } & y & \text { el } & \text { Caribe. }\end{array}$ www.oei.es/salactsi/CAPITULO 03 Dias Sobrinho.pdf

Dopico, I. (2007). Propuesta de un sistema de metaevaluación para el aseguramiento de la calidad de los procesos de evaluación externa y acreditación de los Programas de Maestría en Cuba. Tesis en opción al grado científico de Doctor en Ciencias de la Educación, Universidad de La Habana, Cuba. Recuperado de http://revistas.mes.edu.cu/elibro/tesis/ciencias-de-laducacion/9789591607881.pdf/view 
Revista Gestión de la Educación, Vol.1, №2, pp.35-66, ISSN 2215-2288, julio-diciembre, 2011

Espinoza, O. y González, L. E. (Julio, 2006). Procesos universitarios dinámicos: el modelo de gestión de la calidad total. Revista Calidad en la Educación, 24, 17-34.

Recuperado de http://www.cned.cl/public/secciones/seccionpublicaciones/doc/53/cse articulo496.p $\underline{\mathrm{df}}$

Espinoza, O. y González, L.E. (2008). Calidad de la Educación Superior en América Latina y el Caribe: concepto y modelos. Recuperado de http://www.cres2008.org/upload/documentosPublicos/tendencia/Tema03/

Fernández L., N. (Mayo-agosto, 2004). Hacia la convergencia de los sistemas de educación superior en América Latina. Revista Iberoamericana de Educación: calidad y acreditación universitaria 35, 39-71. Recuperado de http://www.rieoei.org/rie35a02.htm

Garbanzo V., G. (2007). Calidad y equidad de la Educación Superior pública. Aspectos por considerar en su interpretación. Revista Educación 31(2), 11-27. Recuperado de http://redalyc.uaemex.mx/pdf/440/44031202.pdf

Ginés, J. (Mayo-agosto, 2004). La necesidad del cambio educativo para la sociedad del conocimiento Revista Iberoamericana de educación, 35, 13-37. Recuperado de http://www.rieoei.org/rie35a01.htm

Global University Network for Innovation [GUNI]. (2009). La universidad socialmente responsable: una manera de ser en News. Recuperado de http://www.gunirmies.net/news/detail.php?id=1125

González, L. y Ayarza, H. (julio-setiembre, 2006). Acreditación en la Educación Superior en la Región Latinoamericana y del Caribe. Boletín CINDA, N 110. Recuperado de http://www.cinda.cl/download/boletin/b110.pdf

Jacoby, K. (2008). "La teoría del proyecto (Marco Lógico, Cadenas de Impacto)". Seminario de Postgrado Programa de Capacitación en Evaluación de Impactos, Universidad de Costa Rica, San José, Costa Rica.

Jacoby, K. y Mora, D. (2008). Prácticas y ejercicios “La teoría del proyecto (Marco Lógico, Cadenas de Impacto)". Programa de Capacitación en Evaluación de Impactos. 
Revista Gestión de la Educación, Vol.1, №2, pp.35-66, ISSN 2215-2288, julio-diciembre, 2011

Seminario de Postgrado impartido en la Escuela de Administración Pública de la Universidad de Costa Rica, San José, Costa Rica.

L’Ecuyer, J. (Diciembre, 1994). Evaluación y acreditación. Ponencia presentada en el Seminario Taller Centroamericano "El ejercicio de profesor universitario y reconocimiento de estudios universitarios en Centroamérica", Universidad de Costa Rica, San José, Costa Rica.

L' Ecuyer, J. (1994). Garantizar la calidad en un mundo en evolución. La educación: revista interamericana de desarrollo educativo, 38 (118). Recuperado de http://www.educoas.org/portal/bdigital/contenido/laeduca/laeduca 118/investigacio n1/index.aspx?culture=fr\&navid $=107$

Lemaitre, M. J. (2007). Aseguramiento de la calidad de la Educación Superior en Iberoamérica. Ponencia del Seminario Internacional La Educación Superior: antecedentes y perspectivas. Recuperado de http://www.cedus.cl/files/Lemaitre.pdf

Lemaitre, M. J. (2007). Una mirada actual al desarrollo de procesos de aseguramiento de la calidad. Recuperado de http://www.inqaahe.org/admin/files/assets/subsites/1/documenten/1231337782 un a-mirada-actual-al-desarrollo-de-procesos-de-aseguramiento-de-la-calidad[1].pdf

López, F. (2006). Responsabilidad social de la universidad en el escenario mundial actual. Recuperado http://dali.uao.edu.co:7777/pls/portal/docs/PAGE/UNIAUTONOMA INVESTIGACI ONES/ OF DI\%2001/GRUPOS/CATEDRA/DOCUMENTOS/RESP SOCIAL DOC $\underline{\text { PDF }}$

Martínez M., M. (Setiembre-diciembre, 2006). Formación para la ciudadanía y educación superior. Revista Iberoamericana de educación, 42. Recuperado de http:/ /www.rieoei.org/rie42a05.pdf

Montero R., E. (Enero-junio 2004). Marco conceptual para la evaluación de Programas de Salud. Población y Salud en Mesoamérica, (1) 2, 1-18. Universidad de Costa Rica, San José, Costa Rica. Recuperado de http://redalyc.uaemex.mx/src/inicio/ArtPdfRed.jsp?iCve=44601206 
Revista Gestión de la Educación, Vol.1, №2, pp.35-66, ISSN 2215-2288, julio-diciembre, 2011

Nebelung, M. (2004). El sistema de monitoreo y generación de impacto de la GTZ. Recuperado de la base de datos del Curso Evaluación de Impactos, Universidad de Costa Rica. San José, Costa Rica.

Oliva, A. (2008). Aprehensión filosófica de la evaluación de impacto y su determinación. Tesis en opción al grado científico de Doctor en Ciencias Filosóficas, Universidad de la Habana, Cuba. Recuperado de http://revistas.mes.edu.cu/elibro/tesis/ciencias-filosoficas/9789591607812.pdf/view

OREALC/UNESCO. (2008). Reflexiones en torno a la evaluación de la calidad educativa en América Latina $y$ el Caribe. Recuperado de http://unesdoc.unesco.org/images/0017/001776/177648S.pdf

Pires, S. y Lemaitre, M. J. (2008). Sistemas de acreditación y evaluación de la Educación Superior en América Latina y el Caribe. Recuperado de http://www.oei.es/salactsi/CAPITULO 08 Pires.pdf

Quintero, V. (2007). Gestión y evaluación de la gestión universitaria. Ponencia en IX Congreso Iberoamericano de Extensión Universitaria. Colombia.

Rama, C. (2006). La tercera reforma de la Educación Superior en América Latina y el Caribe: masificación, regulación e internacionalización. La metamorfosis de la educación superior. Informe sobre la Educación Superior en América Latina y El Caribe 2000-2005. Recuperado de http://mt.educarchile.cl/mt/ijbrunner/archives/2006/07/iesalc informe sobre la edu cac.html

Rama, C. (2008). Las tendencias de la educación superior en América Latina. Recuperado de http://www.claudiorama.name/archivos/publicaciones/112431a158ecb9792a797afe 6ea4bd19.doc

Sebastián, J. (2003). La dimensión internacional en los procesos de evaluación y acreditación de la Educación Superior. Recuperado de http://www.cna.gov.co/1741/articles-186502 doc academico9.pdf

Silva, C. (2006). Aseguramiento de calidad en gestión de instituciones de Educación Superior: expectativas y desafíos. Calidad en la educación, 24, 117-132. 
Revista Gestión de la Educación, Vol.1, №2, pp.35-66, ISSN 2215-2288, julio-diciembre, 2011

Recuperado

de http://www.cse.cl/public/Secciones/seccionpublicaciones/doc/53/cse articulo500.pdf

Simoneau, R. (2002). La evaluación institucional: conceptos teóricos. Academia de Evaluación Institucional. Recuperado de http://valle-mexico.pm.org/evins/

Sistema Nacional de Acreditación de la Educación Superior [SINAES]. Manual de acreditación oficial de carreras de grado del Sistema Nacional de Acreditación de la Educación Superior. Recuperado de http://www.sinaes.ac.cr/manual guias/manual oficial acreditacion vf feb2010.pdf

Sol, R. (2003). Reformas y tendencias de cambio en la Educación Superior y sus instituciones en Centroamérica. Observatorio de la Educación Superior en América Latina y el Caribe, IESALC/UNESCO. Recuperado de http://www.inca-network.org/public documents/Reforma ES CA IESAL

Suárez, J. y Comboni, S. (2007). La calidad de la educación en el discurso políticoacadémico en México. Reencuentro 50, 60-72. Recuperado de http://redalyc.uaemex.mx/redalyc/src/inicio/ArtPdfRed.jsp?iCve=34005009

Tünnermann, C. (2000). Calidad, evaluación institucional, acreditación y sistemas nacionales de acreditación. Instituto Latinoamericano de Educación para el Desarrollo. Recuperado de http://www.ilaedes.org/documentos/articulos/evaluacionyacreditacion.pdf

UNESCO. (1998). Declaración Mundial sobre la Educación Superior en el siglo XXI: Visión y acción. Conferencia Mundial sobre Educación Superior. Recuperado de http://www.unesco.org/education/educprog/wche/declaration spa.htm

UNESCO. (2009). La nueva dinámica de la educación superior y la investigación para el cambio social y el desarrollo. Informe de la Conferencia Mundial sobre Educación Superior. Recuperado de http://www.unesco.org/education/WCHE2009/comunicado es.pdf

Zapata, G. y Tejeda, I. (2009). Impactos del aseguramiento de la calidad y acreditación de la Educación Superior: consideraciones y proposiciones. Calidad en la educación, 31, 192-209. Recuperado de http://www.cned.cl/public/secciones/seccionpublicaciones/doc/64/cse articulo839.pdf 